\title{
CORRIGENDA TO VOLUME 105
}

Page 85, penultimate line to read: "were suspended, not merely for a few days, to be followed shortly in 2 by the'

Page 86, third line. For 'Three' read 'There'.

Part 1, back cover and Part 3, contents list of volume. Seventh item: For '1976' read '1977'.

Page 209, third line. For 'Hutchinson' read 'Heinemann'.

Page 301, fourth line of fourth paragraph of Mr Hey's remarks. For 'integrals' read 'intervals'.

Page 304. Mr Carter's remarks should begin:

'I think the definition of 'provision' in the Companies Act is easy to understand: it is any amount set aside to meet a known liability', the amount of which cannot be determined with substantial accuracy. A 'reserve' is anything which is not a 'provision'!

Page 305. Ninth line of first paragraph of Professor Cox's remarks. For 'Coshy' read 'Cauchy'.

Page 308. Insert before the author's reply:

The President (Mr C. M. O'Brien), proposing a vote of thanks: I apologize for the paper being available only a very short time in advance of the meeting. However, this has meant that the discussion has concentrated more on the broad aspects of the subject rather than on the detail and I think this is no bad thing. Now that the paper is available, those concerned may go into its details and try out the techniques with their own data. I am sure that it will be a happy hunting ground for the General Insurance Research Group in the months to come. The paper does indicate clearly that there is a very long way to go before we can claim any acceptable and scientific method, a fully coherent method of reserving which would also apply, mutatis mutandis, to the question of rate setting.

Page 309, fourth line. For B. Beard read R. E. Beard. 